Rev. Elev. Méd. vét. Pays trop., 1972, 25 (1): 79-83

\title{
Essais cliniques du Nitroxynil dans le traitement de l'ankylostomose des chiens
}

\author{
par P. M. TRONCY $(*)$ et J. J. DELAITRE $\left({ }^{* *}\right)$
}

\begin{abstract}
RESUME
Les auteurs décrivent l'expérimentation dans les conditions de la clinique du Nitroxynil (Dovenix) contre les Ankylostomes du chien. L'essai a porté sur 30 sujets. Les résultats sont estimés bons dans leur ensemble, quoiqu'une défaillance ait été enregistrée.
\end{abstract}

Le Nitroxynil $\left({ }^{1}\right)$ est un anthelminthique d'action désormais classique sur Fasciola sp. et en outre réputé actif sur des Nématodes: Haemonchus sp., Bunostomum sp. et Ancylostoma caninum.

Au Tchad, l'ankylostomose des carnivores est très fréquente et meurtrière. Aussi l'activité du Nitroxynil contre les Ankylostomes a-t-elle retenu l'attention, et ce médicament a été testé dans les conditions courantes de la clinique. Ces essais ont été effectués pendant le premier semestre de l'année 1971.

\section{MATERIEL ET METHODES}

\section{SELECTION DES MALADES}

Elle s'est faite très simplement sur les animaux "tout venant " présentés à la clinique vétérinaire de Fort-Lamy.

Tous les chiens suspects d'ankylostomose furent soumis à un examen coprologique qua-

(*) I.E.M.V.T. Laboratoire de Recherches vétérinaires de Farcha, B.P. no 433, Fort-Lamy (Tchad).

(**) Institut d'Enseignement Zootechnique et Vétérinaire d'Afrique Centrale, Fort-Lamy (Tchad).

(1) Commercialisé en France par les laboratoires SPECIA sous le nom de DOVENIX. litatif et quantitatif, selon la méthode simplifiée de E. BRUMPT qui, sans prétendre à la précision, est suffisante pour obtenir une approximation du degré des infestations. On eut beaucoup de difficultés pour suivre l'effet du traitement chez les chiens des particuliers : ou bien ceux-ci présentaient leurs chiens pour lesquels on faisait une coprologie, puis ne revenaient plus, ou bien ne revenaient que pour la première injection, ou bien disparaissaient après la seconde.

En pratique, ce sont surtout les pensionnaires du chenil de la base aérienne de Fort-Lamy qui ont fait l'objet d'une observation suivie. On eut à y déplorer deux épidémies d'ankylostomose, la première à la fin de l'année 1970 rebelle aux traitements classiques - fut traitée avec succès par le Nitroxynil. La seconde, en mai-juin 1971, parut tout d'abord surprenante car en pleine saison sèche. On put craindre un simple réveil d'une infestation provisoirement masquée par le précédent traitement, mais une observation complète des lieux permit de s'apercevoir qu'il s'agissait d'une réinfestation réalisée à partir de l'eau de nettoyage des cages, dont l'écoulement à ruisseau perdu derrière les bâtiments de l'établissement, entretenait une végétation herbeuse où aimaient venir gambader les chiens au cours de leur promenade quotidienne. 


\section{Remarque}

Une enquête récente a montré que l'on trouve au Tchad Ancylostoma (Ancylostoma) caninum (Ercolani, 1859) dans les $2 / 3$ des infestations, et Ancylostoma (Afrancylostoma) braziliense (De Faria, 1910) dans les autres cas. Uncinaria stenophala (Railliet, 1884) n'a, à ce jour, jamais été rencontré.

\section{TRAITEMENT DES MALADES}

La clinique vétérinaire reçut un lot d'échantillons spécialement dosés pour être utilisés chez les carnivores (ampoules de $5 \mathrm{ml}$, à $2 \mathrm{p}$. 100 de principe actif). Plus tard, l'essai fut poursuivi avec la solution courante à 25 p. 100 de principe actif, diluée à 2 p. 100 avant l'emploi, avec de l'eau distillée.

Toutes les administrations ont été faites par voie parentérale et, quelle que soit la voie choisie, toujours injectées en deux endroits différents du corps de l'animal.

Dans 5 cas, seule la voie sous-cutanée a été utilisée; dans 7 cas, on a associé la voie souscutanée et la voie intramusculaire (pour chaque intervention, une demi-dose par voie sous-cutanée, une demi-dose par voie intramusculaire). Enfin, dans 18 cas, seule la voie intramusculaire a été employée.

\section{DOSES}

Dans tous les cas, $10 \mathrm{mg}$ par $\mathrm{kg}$ de poids vif, renouvelés à 48 heures d'intervalle.

\section{CONTROLE D'EFFICACITE}

1. Les propriétaires furent sollicités pour vérifier l'absence ou la présence de vers dans les fèces, dans les jours suivant les interventions; ils ne signalèrent jamais avoir observé l'expulsion de Nématodes.

2. L'amélioration de l'état général des animaux fut un important critère d'efficacité du traitement.

3. 7 à 10 jours après la fin du traitement, une coprologie de contrôle fut pratiquée pour évaluer la persistance éventuelle du parasitisme.

\section{RESULTATS}

Les résultats sont synthétisés dans le tableau de la page suivante.

\section{COMMENTAIRES}

1. Dans tous les cas, l'amélioration de l'état des animaux traités a été rapide et spectaculaire : dès le lendemain du traitement, un mieux était observé.

\section{Incidents}

a) le chien $\mathrm{n}^{\circ} 4(+++++)$ est mort après la $2^{\mathrm{e}}$ injection. Cet animal était dans un état général extrêmement bas, avec les symptômes habituels de l'ankylostomose; l'infestation (432 œufs par gramme) était moyenne pour les conditions locales.

Le traitement fut bien toléré, mais la mort survint le lendemain de la $2^{\mathrm{e}}$ injection.

Le propriétaire refusa catégoriquement l'autopsie. L'altération de l'état général ne fut observée chez aucun autre animal avec une telle gravité. En l'absence de preuves péremptoires, il semble possible d'envisager la possibilité d'une association de deux affections. Il existe en effet au Tchad une maladie relativement fréquente chez les chiens, dont l'agent est encore mal connu; elle est désignée par certains auteurs sous le nom de " Pancytopénie tropicale ", et certains symptômes (adénites, épistaxis chez le Berger Allemand) évoquent l'ankylostomose. Le Nitroxynil a-t-il contribué à la mort de l'animal ? Il est impossible de répondre. Avec l'expérience que donne la pratique de la clinique, on pense que ce Berger Allemand, traité ou non, serait mort de toute façon.

b) sur les chiens $1,2,5,6$, 9 et 10 , des œdèmes se sont développés aux points d'injection.

- Chiens 1 et 3 : Les injections ont été faites par voie sous-cutanée uniquement, et les réactions ont été très fortes. Chez le $\mathrm{n}^{10} 1$, l'œdème, volumineux, s'est développé à l'endroit de la $2^{\text {e }}$ injection, il s'est rapidement résorbé. Chez le $\mathrm{n}^{0} 3$, l'œdème est apparu à $l^{\prime}$ endroit de la $1^{\text {re }}$ injection; devenu très dur, il persista longtemps, avec tendance à s'organiser. 




s.c. = sous cutanée; $\mathrm{i}$.m. = intramusculaire. 
- Chiens 5, 6, 9 et 10 : Chez tous ces sujets, on a pratiqué une double injection (voie sous-cutanée et voie intramusculaire) à chaque intervention. Chaque fois un cedème plus ou moins net, et vite résorbé (environ 3 jours) s'est développé au point de la $2^{\mathrm{e}}$ injection souscutanée. Statistiquement, l'apparition d'œè̀me en relation avec l'injection sous-cutanée, et l'absence d'œdème après injection intramusculaire, sont significatives.

On notera que tous ces sujets étaient des Boxers. Il n'y eut jamais d'incidents avec les chiens des autres races. A la suite de ces incidents, toutes les injections furent pratiquées par voie intramusculaire.

\section{Examens incomplets}

On observera que les chiens $\mathrm{n}^{\text {os }} 12,13,16$ et 30 ne se sont pas présentés aux contrôles. On peut cependant les inclure dans cette série d'observations, car on a pu savoir par la suite que leur état était redevenu tout à fait normal à la suite de l'intervention.

Chez les sujets $\mathrm{n}^{\text {«s }} 15$ et 29 , seul le diagnostic clinique d'ankylostomose a été posé, à l'exclusion du diagnostic coprologique. Dans les deux cas, l'amélioration immédiate de l'état général a confirmé a posteriori le diagnostic.

\section{Traitements systématiques}

Les chiens $\mathrm{n}^{\text {ws }} 10,11,27$ et 28 , bien que négatifs à l'examen coprologique, ont été traités par le Nitroxynil. II s'agissait de chiens du chenil de la base aérienne, soignés en raison du degré d'infestation de l'effectif. Chez ces
4 chiens, un contrôle de routine a également été pratiqué; ce contrôle n'était pas vain, puisque, avec le chien $n^{\circ} 10$, a été mise en évidence la seule défaillance enregistrée avec le médicament.

Il faut préciser que le traitement du chien $\mathrm{n}^{*} 10$ (ainsi que ceux des chiens $\mathrm{n}^{\text {os }} 5,6,7,8,9$, et 11) n'avait été effectué qu'un mois après l'examen coprologique. L'expérimentation avait, en effet, été momentanément interrompue à la suite des incidents survenus avec les chiens 1,3 et 4 . Il est possible que le sujet $\mathrm{n}^{0} 10$ se soit infesté entre le 11 janvier et le 8 février 1971.

\section{CONCLUSIONS}

Les résultats obtenus avec le Nitroxynil par voie parentérale dans le traitement de l'ankylostomose des carnivores sont dans leur ensemble satisfaisants.

Pour cette expérimentation réalisée sur 30 animaux dans les conditions de la clinique, on a utilisé la dose de $10 \mathrm{mg}$ de produit actif par $\mathrm{kg}$ d'animal, dose renouvelée après 48 heures. Seule la voie intramusculaire n'a provoqué aucune réaction locale.

Une défaillance du médicament a été enregistrée chez le sujet $\mathrm{n}^{\circ} 10$.

Au cours de l'expérimentation, un chien est mort le lendemain de l'intervention; mais le Nitroxynil ne peut formellement être mis en cause, compte tenu des commémoratifs et de la pathologie locale.

\section{SUMMARY}

\section{Clinical trials of Nitroxynil in the
treatment of dog ankylostomiasis}

The authors describe the experimentation, in clinical conditions, of the Nitroxynil against Hookworms of dogs. The test was done with 30 animals. The results are good in the whole, although a failing had been recorded.

\section{RESUMEN}

Ensayos clinicos del Nitroxynil para el tratamiento de la anquilostomiasis del perro

Los autores describen la experimentación, en las condiciones clínicas, del Nitroxynil (Dovenix) contra los anquilostomas del perro.

Se utilizaron 30 animales durante el ensayo.

Se estimar los resultados buenos en su conjunto, aunque se ocurro un fracaso. 


\section{BIBLIOGRAPHIE}

LUCAS (J. M. S.), Le iodo-3 hydroxy-4 nitro-5 benzonitrile (Nitroxynil - $10.755 \mathrm{M}$ et B - 16.886 R.P.). I. Activité dans la distomatose expérimentale du lapin, du mouton et du veau, $C a h$. Méd. vét., 1967,36 (6) : 169-179.
EUZEBY (J.), GARCIN (Cl.) et GROSJEAN (N.), Activité anti-ankylostomienne d'un dérivé halogéno-nitré du phénol, le iodo-3 hydroxy-4 nitro-5 benzonitrile (Nitroxynil) : étude préliminaire, Bull. Soc. Sci. vét. Lyon, 1968, 70 (6) : 481-487. 